\title{
Treaties, conventions and other international instruments
}

Anti Counterfeiting Trade

Agreement (ACTA) $\mathbf{4 1 6}$

Berne Convention, 1886 as amended

$1,8,9-13,14,15,17,18,19,20$,

25, 27, 39, 58, 404

Art $2 \quad 20$

Art 2(1) $\mathbf{1 2}$

Art 2(5) 20, 41

Art 2(7) 12, 181

Art 311

Art 5(1) 11

Art 5(2) $\quad 11$

Art 5(3) 11

Art 6bis 13, 17, 20

Art $7 \quad 17$

Art 7(1) 13

Art 7(4) 21, 181, 203

Art 7(8) $\mathbf{1 2}$

Art $8 \quad \mathbf{1 2}$

Art 9 12, 20

Art 9(2) 12, 18

Art $10 \quad 12$

Art 10bis $\mathbf{1 2}$

Art $11 \quad 21$

Art 11(1) 12

Art 11(2) 12

Art 11bis 12, 21

Art 11ter 12, 21

Art $12 \mathbf{1 2}$

Art 14 12, 14

Art 14(1)(i) 20

Art 14bis (2) $\mathbf{2 0}$

Art 14ter (2) 12
Paris Act, 1971, Arts 1-21 17, 20

Appendix 20

Brussels Convention 146, 201, 275

Budapest Treaty on the international recognition of the deposit of micro-organisms for the purposes of patent procedure, 1977

Art $7 \quad 166$

Caracas Convention, $1911 \mathbf{1 3}$

Community Patent Convention, 1975 149, 150

Art 2(2) 149

Convention on Biological Diversity (CBD), 1992, UN 70, 82, 83, 85-8, 163

Preamble 86

Art $1 \quad 86$

Art $2 \quad 86$

Art $3 \quad 87$

Art $6 \quad 87$

Art $15 \quad 87$

Art $16 \quad 87$

Art 16(5) 87

Art $20 \quad 86$

Cartagena Protocol on Biosafety, 2000 87, 88

Convention on International

Exhibitions, Paris $1928 \quad 106$

EC Convention on the Law

Applicable to Contractual

Obligations (Rome I) 156

European Economic Area (EEA) 
Agreement, 2 May 1992 OJ 1994

L1

Art 65(2) 360

Prot 28, Art $2 \quad \mathbf{3 6 0}$

Annex XVII 360

European Convention on Human

Rights (ECHR) 162, 411

European Patent Convention (EPC),

$19735, \mathbf{7 0}, \mathbf{7 2}, \mathbf{7 3}, \mathbf{9 1}, \mathbf{9 2}, 132$

Part X (Arts 150-3) 75

Art $14 \quad 96$

Art $52 \quad 109$

Art 52(2)(c) 93

Art 52(4) 110, 117, 132

Art 54(5) 109, 110

Art $57 \quad \mathbf{1 3 2}$

Art 69(1) 102

European Patent Convention (EPC)

1973, Rules

r 23b 118, 119, 127, 128

r 23b(5) 131

r 23c 128

r 23c(b) 130

r 23c(c) 132

r 23d 134, 138

r 23d(c) 139

r $23 \mathrm{~d}(\mathrm{~d}) \quad \mathbf{1 4 0}$

r $23 \mathrm{e} \quad \mathbf{1 1 9}, 122$

European Patent Convention, 2000

(EPC 2000) 5, 70, 72, 73,

92-100, 125, 149, 153, 155, 161,

162

Art 294

Art 4(a) 94

Art $14 \quad 96$

Art 14(4) 96

Art $21 \quad 101$

Art $22 \quad 101$

Art $33 \quad 94$

Art 33(1)(b) 94

Art 51(1) $\quad 106$

Art 51(1)(a) 106
Arts 52-57 100

Art 52 92, 109, 118, 119, 124, 125

Art 52(1) 92, 93, 99, 104, 105, $111,117,119,120,123$

Art 52(2)-53 71, 118 Art 52(2) 104, 118, 119, 120 ,

$121,123,124,126$

Art 52(2)(c) 122, 123, 126

Art 52(3) $\quad 123$

Art 52(4) $\quad 109$

Art 53 104, 110, 118

Art 53(a) 133-41, 164

Art 53(b) 127-32, 163

Art 53(c) 110, 117, 132-3

Art 54 105-11

Art 54(1) 105, 108, 110

Art 54(2) 105, 110, 111

Art 54(3) $\quad \mathbf{1 0 5}$

Art 54(4) $\quad 109$

Art 54(5) $\quad \mathbf{1 0 9}$

Art 55(1) 106

Art 55(1)(b) $\mathbf{1 0 6}$

Art 56 111-17

Art 57 117-18

Art $58 \quad 95$

Art $59 \quad 95$

Art $60 \quad 95$

Art 60(3) 95

Art $61 \quad 96$

Art 63 92, 94, 158

Art $64 \quad 94$

Art 64(2) 97

Art 64(3) 102, 142

Art 65 100, 155

Art $66 \quad 98$

Art $67 \quad 99$

Art 67(2) $\mathbf{1 5 5}$

Art 67(3) 155

Art $68 \quad \mathbf{1 0 0}$

Art 69 93, 102-4

Protocol on Interpretation of Art

69 93, 102-4, 143 
Art 69(1) $\mathbf{1 0 2}$

Art 70(3) $\mathbf{1 5 5}$

Art 70(4) 155

Arts 71-3 100

Art 74 102, 142

Art $75 \quad 95$

Art $78 \quad 96$

Art $79 \quad 96$

Art $80 \quad 98$

Art $82 \quad 98$

Art 83 104, 141

Art $84 \quad 96$

Art $86 \quad \mathbf{1 0 0}$

Art $87 \quad 98$

Art 87(1) 96

Art 87(4) 96

Arts 88-9 98

Art $90 \quad 98$

Art $92 \quad 98$

Art $93 \quad 99$

Art 9499

Art $97 \quad \mathbf{1 0 0}$

Art $99 \quad \mathbf{1 0 0}$

Art $100 \quad \mathbf{1 0 0}$

Art 100(b) 104, 141

Art $101 \quad 101$

Arts 105a-c 101

Art $106 \mathbf{1 0 1}$

Art $112 \mathbf{1 0 1}$

Art 112a 101

Art 112(1)(b) $\mathbf{1 2 4}$

Art $121 \quad 93$

Art $122 \quad 93$

Art 123(2) 99

Art 123(3) 101

Art $138 \quad \mathbf{1 0 2}$

Art 138(1) 157

Art 138(1)(a) $\mathbf{1 0 5}$

Art $139 \quad \mathbf{1 0 2}$

Art 149(a) 94

Protocol on Jurisdiction and the

Recognition of Decisions in
Respect of the Right to the

Grant of a European Patent 95

EPC 2000 Rules of Implementing

Regulations 118, 119, 121, 127

r 6(1) 96

r 6(2) 96

rr 14-18 96

r $26118,127,128$

r 26(5) 131

r $27 \quad \mathbf{1 2 8}$

r 27a 121

r 27(b) 130

r 27(c) $\quad 132$

r $28 \quad \mathbf{1 3 4}, \mathbf{1 3 8}$

r 28(c) 139

r 28(d) $\quad 140$

r $29 \quad \mathbf{1 2 2}$

r $30 \quad 97$

r $31 \quad 97$

rr 32-34 97

r $35 \quad 95$

r 42(1)(c) $\quad 115$

r $44 \quad 98$

r 47(2) $\quad 115$

r $51 \quad \mathbf{1 0 0}$

r 6198

r $62 \quad 98$

r $64 \quad 98$

r $65 \quad 99$

r 70(1) 99

r $71 \quad 99$

r $72 \quad 95$

r $76 \quad \mathbf{1 0 0}$

r $80 \quad \mathbf{1 0 1}$

General Agreement on Tariffs and Trade (GATT) 17, 25, 26

Hague Agreement $\mathbf{5}$

Geneva Act $\mathbf{1 8 4}$

International Convention for the

Protection of New Varieties of

Plants, 1961 (UPOV) 129, 130 
Art 2(1) $\mathbf{1 2 9}$

Lisbon Agreement for the Protection of Appellations of Origin and their International Registration, $1958 \quad 291$

Art $2 \quad 291$

Art 3291

Locarno Convention 187

London Agreement, $2000 \quad \mathbf{1 4 2}$,

\section{3-4}

Lugano Convention 146

Luxembourg Convention 149

Madrid Agreement, $1891 \quad \mathbf{5 , 2 1 2}$,

214-16, 217, 254, 291, 331

Art 1(2) 215

Art 1(3) 215

Art 3(4) 215

Art 4(1) 215

Art 5(1) 215

Art 5(2) 215

Art 6(2) 216

Art 6(3) 216

Madrid Protocol, 1989 (Protocol

Relating to the Madrid

Agreement Concerning the

International Registration of

Marks) 212, 216-18, 254

Art $2 \quad 217$

Art 3(1) 217

Art 3(4) $\quad 217$

Art $4 \quad 217$

Art 5(1) 217

Art 5(2)(b) $\quad 217$

Art 5(2)(c) $\mathbf{2 1 8}$

Art $6 \quad 218$

Art 6(3) 218

Art $7 \quad 218$

Art 9quinques $\mathbf{2 1 8}$

Art 9sexies $\mathbf{2 1 8}$

Art 14(1) 217

Montevideo Convention, 1889

Nice Agreement Concerning the
International Classification of

Goods and Services for the

Purposes of Registration of

Marks, $1957 \quad 222$

Pan American Conventions of 1902,

1906, 1910, $1928 \mathbf{1 3}$

Paris Convention, 1883 as revised

$1,17,74-5,95,98,181,187$,

212, 213-14, 215, 216, 217, 223 ,

290, 331, 404

Art $1 \quad \mathbf{7 5}$

Art 1(2) 291

Art 2 74, 181

Art $3 \mathbf{7 4}$

Art 4 74, 214

Art 4 bis $\mathbf{7 4}$

Art 4C(1) $\quad 181$

Art 6bis 214, 219

Art 6ter 200, 253

Art 6quinques 214, 215

Art 6sexies 214

Art 6a 254

Art 6(1) $\mathbf{2 1 3}$

Art 6(2) $\mathbf{2 1 4}$

Art 6(3) $\mathbf{2 1 4}$

Art 7bis $\mathbf{2 1 4}$

Art 9291

Art $10 \quad 291$

Art 10bis 214, 292

Art 10(2) 291

Art $13 \quad \mathbf{7 5}$

Art $14 \quad 75$

Patent Cooperation Treaty (PCT)

70, 73, 75-7, 89, 91, 95

Ch II $\mathbf{7 6}$

Arts 3-8 77

Art 17(2)(a)(i) $\mathbf{7 6}$

Rule $12 \quad \mathbf{7 6}$

Rule $39 \quad \mathbf{7 6}$

Patent Law Treaty (PLT), 2000,

WIPO 88-91

Art $5 \quad 88$ 
Art $6 \mathbf{8 8}$

Rio Declaration on Environment and

Development

Principle $15 \mathbf{8 8}$

Rome Convention for the Protection of Performers, Producers of

Phonograms and Broadcasting

Organisations, 1961 14-17, 18,

22, 23, 38, 40

Art $2 \quad 15$

Art 3(a) 15

Art 3(b), (c) 16

Art $4 \quad 15$

Art $5 \quad 15$

Art $6 \quad \mathbf{1 5}$

Art 7 15, 22

Art $9 \mathbf{1 5}$

Art $10 \quad \mathbf{1 5}$

Art 11 16, 23

Art 12 16, 32

Art $13 \quad 16$

Art 14 15, 16

Art 15(1) $\mathbf{1 6}$

Art 15(2) 16

Art $16 \quad 16$

Singapore Treaty on the Law of

Trademarks 219

Trademark Law Treaty (TLT), 1994

218-19

TRIPS: Agreement on trade-related

aspects of intellectual property

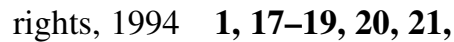

$23,26,70,77-9,82,83,85,92$,

$161,163,173,213,223,292-4$,

295, 404-6

Art 298

Art $6 \mathbf{3 7 2}$

Art 9.1 17, 20, 21

Art 9.2 17, 20

Art 10.1 18, 20

Art 10.2 19, 20

Art $11 \quad 19$
Art $12 \quad 17$

Art $13 \quad \mathbf{1 8}$

Art $14 \quad 20$

Art $14.1 \quad \mathbf{1 8}$

Art $14.2 \quad \mathbf{1 8}$

Art $14.3 \quad \mathbf{1 8}$

Art 14.4 18, 19

Art 14.5 15, 18

Art $14.5 \quad 18$

Arts 15-21 219

Art $15 \quad 219$

Art $16.2 \quad 219$

Art $16.3 \quad 219$

Art $17 \quad \mathbf{2 2 0}$

Art $18 \quad \mathbf{2 2 0}$

Art $19 \quad \mathbf{2 2 0}$

Art 19(1) 272

Art $21 \quad 220$

Art 22(1) 292

Art 23 292, 293

Art 23(4) 292

Art $27.1 \quad \mathbf{7 7 , 9 3}$

Art 27.2 77, 134

Art 27.3(a) 77

Art 27.3(b) 78, 82-5, 173

Art $28 \quad \mathbf{7 8}$

Art 29(1) 78

Art $30 \quad \mathbf{7 8}$

Art $31 \quad 79$

Art 31(f) 80, 81

Art 31 bis $\mathbf{8 1}$

Art 33 78, 79

Art $41 \quad \mathbf{4 0 5}$

Art $42 \quad \mathbf{4 0 5}$

Art 43(1) $\mathbf{4 0 5}$

Art 44(1) $\mathbf{4 0 5}$

Art 45(1) $\mathbf{4 0 6}$

Art 45(2) $\mathbf{4 0 6}$

Art $46 \quad \mathbf{4 0 6}$

Art $47 \quad \mathbf{4 0 6}$

Art 50(1)(a) $\mathbf{4 0 5}$

Art 50(1)(b) $\mathbf{4 0 6}$ 
Art 50(2) 406

Arts 50(3)-(7) 406

Art $51 \quad 406$

Arts 52-60 406

Art $61 \quad \mathbf{4 0 6}$

Doha Declaration, 2001

79-81, 82, 84, 85, 292, 293, 372

Protocol amending the TRIPS

Agreement, $2005 \mathbf{8 1}$

Universal Copyright Convention

1952 (revised 1971, Paris)

13-14

Art III 14

Art IV 14

UPOV Convention 1961, as revised

172-4

Art $2 \quad 173$

Art $4 \quad 173$

Arts 5-9 173

Art $11 \quad \mathbf{1 7 3}$

Art $14 \quad 173$

Art $15 \quad 173$

Art 17(1) 173

Art $19 \quad \mathbf{1 7 3}$

Venice Resolutions, 2005, 2006

147

Vienna Agreement Establishing an

International Classification of the

Figurative Elements of Marks,

$1973 \mathbf{2 2 3}$

Vienna Convention on the Law of

Treaties, 1969

Art $31 \mathbf{1 2 5}$

WCT see WIPO Copyright Treaty

(WCT)

WIPO Copyright Treaty (WCT)

$$
\text { 19-21, 23, } 50
$$

Preamble 20

Art $1 \quad 20$
Art 1(4) 20

Art $2 \quad \mathbf{2 0}$

Art $4 \quad 20$

Art $5 \quad 20$

Art $6 \quad 20$

71, Art $7 \quad 20$

Art 7(1) $\quad \mathbf{2 0}$

Art 7(2) $\quad 20$

Art 7(3) 20

Art $8 \quad 21$

Art $9 \quad 21$

Art $10 \quad 21$

Art 11 21, 55

Art $12 \quad 21$

Art $14 \quad 21$

Art $17 \quad 19$

WIPO Internet Treaties (WIPO

Copyright Treaty (WCT) and

WIPO Performances and

Phonograms Treaty (WPPT)) 5,

19-23, 26, 50

WIPO Patent Cooperation Treaty $\mathbf{8 3}$

WIPO Patent Law Treaty $\mathbf{9 2}$

WIPO Performances and

Phonograms Treaty (WPPT),

2002 21-3, 50

Preamble 21

Art 22

Art $5 \quad 22$

Art $6 \quad 22$

Arts 7-10 22

Arts 11-14 22

Art $15 \quad 23$

Art $16 \quad \mathbf{2 3}$

Art $17 \quad \mathbf{2 3}$

Art $18 \quad \mathbf{2 3}$

Art $19 \quad \mathbf{2 3}$

Art $20 \quad \mathbf{2 3}$

Art $23 \quad \mathbf{2 3}$

Art $26 \quad 21$ 